\title{
A new free light chain immunoassay shows advantages in the classification and in the follow-up of patients with paraproteinemia compared to a nephelometric assay
}

\author{
Van Helden $\mathrm{J}^{1}$, Evliyaoglu $\mathrm{O}^{2}$, Dreßen $\mathrm{D}^{1}$ and Weiskirchen $\mathrm{R}^{2}$ \\ ${ }^{1}$ Laboratory Diagnostic Center, University Hospital RWTH Aachen, Germany \\ ${ }^{2}$ Institute for Molecular Pathobiochemistry, Experimental Gene Therapy and Clinical Chemistry, University Hospital RWTH Aachen, Germany
}

\begin{abstract}
Background: For screening for monoclonal gammopathies, their prognostic stratification and therapy monitoring, the quantitative determination of free immunoglobulin light chains is a significant component. Nephelometric and turbidimetric test methods have so far been associated with analytical limitations and pitfalls. The new quantitative s-FLC ELISA (Sebia) was tested for its suitability for use in routine clinical diagnostics in an application study in a supraregional laboratory center in Germany.

Materials and Methods: 510 samples in which the free light chains had previously been determined with the Freelite assays (The Binding Site) were compared with the new ELISA. Furthermore, the M-protein peak concentrations from 25 serum protein electrophoresis (SPE) were compared with the results of free light chain measurements of Freelite and Sebia FLC.

Results: Only moderate correlations for the $\kappa$ and $\lambda$ light chains between the two methods, Sebia FLC ELISA and Freelite, were found. The concordance correlation coefficients (CCC) were only $r=0.68$ and $r=0.67$, respectively. There were significant quantitative differences between the two methods, which were particularly pronounced in samples with high FLC concentrations. The agreement between the immune fixation, which is considered the gold standard, and the Sebila FLC determination was $92.6 \%$, but only $83.0 \%$ for the Freelite tests. The Sebia monoclonal FLC concentrations were consistent with the M protein concentrations determined with the SPE. Compared to this, the Freelite monoclonal FLC concentrations were consistently higher. The average overestimation was 10-fold compared to the SPE.

Conclusion: The Sebia FLC Assay proved to be a robust platform for the sensitive and accurate determination of free light chains in serum. Due to the good agreement with the SPE and the conformity to the immunofixation as well as the low rerun rate resulting from the larger measuring range of the Sebia sFLC ELISA, these assays are suitable alternatives for the determination of free light chains for screening on monoclonal gammopathies as well as for the follow-up of patients with multiple myeloma.
\end{abstract}

\section{Introduction}

Since the availability of the serum free light chain (sFLC) assay, the diagnosis, monitoring and prognosis for plasma cell dyscrasias has greatly improved, because the $\kappa / \lambda$ ratio represents a sensitive balance between the two types of light chain [1]. Overexpression of a light chain type by a malignant B-cell clone leads to a shift in the $\kappa$ to $\lambda$ ratio reference range [2] so that it is possible to identify affected patients before the disease has progressed to the extent that BenceJones proteinuria becomes detectable in the urine. This has ultimately led to the inclusion of the determination of FLC in the guidelines for the diagnosis, treatment and follow-up of multiple myeloma [3]. An incorrect determination of FLC can therefore have direct consequences for the outcome of the patient.

However, the nephelometric and the turbidimetric methods for the determination of FLC are accompanied by a series of analytical limits, pitfalls and technical difficulties [4]. Depending on the lot, an effect can occur with $\kappa$ as well as with $\lambda$.
Because of the unreliability of the lower end of the calibration range of these assays, an analytical gap between $1 \mathrm{mg} / \mathrm{L}-7 \mathrm{mg} / \mathrm{L}$ can occur, which can have a dramatic effect on the calculation of the $\mathrm{k} / \lambda$ ratio. This can lead to an abnormal $\kappa / \lambda$ ratio in healthy individuals and apparently significant changes in the ratio between sequential samples from myeloma patients who are in fact still in remission [5]. Strong discrepancies between the FLC concentrations of Freelite (The Binding Site, Birmingham, UK) and the monoclonal FLC band on serum protein electrophoresis (SPE) were reported [6]. Freelite overestimation can be greater than 10 times and has been attributed

${ }^{\star}$ Correspondence to: Van Helden Josef, Laboratory Diagnostic Center, University Hospital RWTH Aachen, Pauwelsstr. 30, D-52074 Aachen, Germany, Tel +49 2161 81940; E-mail: jvhelden@ukaachen.de

Key words: free light chain, multiple myeloma, nephelometry, turbidimetry, immunoassay, M-protein, immunofixation

Received: October 31, 2019; Accepted: November 07, 2019; Published: November 11, 2019 
Helden VJ (2019) A new free light chain immunoassay shows advantages in the classification and in the follow-up of patients with paraproteinemia compared to a nephelometric assay

to FLC polymerization, which leads to larger immune complexes and greater scattering by nephelometry. FLC concentrations in the near range of the values obtained with SPE were detected by using a new commercially available ELISA test [6]. The quantitative sFLC values obtained with these assays show a better comparability to M- protein concentrations generated by the integration of the paraprotein peaks in the SPE which is listed in the guidelines as a reference for diagnostics, staging and response criteria [1].

Here we present data from a large-scale validation of this observation using the Sebia FLC immunoassay (Sebia, Lisses, France) compared to the Freelite assays performed on BNII nephelometer (Siemens, Eschborn, Germany). The aim of the investigations was to determine whether significant differences occur in a routine collective due to the use of different measurement methods with a consequence for diagnostics or the further course of therapy in patients with multiple myeloma.

\section{Material and Methods}

The study used serum samples from a supraregionally operating routine laboratory in North Rhine-Westphalia, Germany, from different oncological centers for the diagnosis of dysproteinemia or for follow-up.

Comparative analysis of Sebia and Freelite results was done anonymously on the same working day, preventing to draw conclusions on individual subjects. The local ethics committee at the RWTH University Hospital Aachen approved our study (EK158/18). This authority has provided a written statement that we do not require a special ethical vote because the retrospective study is covered by $\$ 6$ of the law for the protection of personal data in the health care system ( $\$$ 6 GDSG NW), which allows data processing for scientific purposes. In the federal state North Rhine-Westphalia this law is still valid even after the introduction of the new EU General Data Protection Regulation (EU-DSGVO) on May 25, 2018.

For the biomarker measurements, a total of 510 serum samples were measured over 18 working days. The FLCs were measured with Sebia FLC ELISA and Freelite (The Binding Site) on BNII nephelometer (Siemens) according to the valid manufacturer's instructions for use. For all samples capillary electrophoresis and immunofixation were available to evaluate the results. The data from the laboratory journal were used to evaluate the rerun rate of BNII nephelometer.

In order to assess the diagnostic performance of the calculated kappa/Lambda quotients, the deviations of the quotients from the reference ranges were compared with the clinical data and the qualitative results of the immune fixation.

For sFLC methods comparison with SPE, sera obtained from patients with measurable involved FLC peaks were analysed on Freelite and Sebia FLC and compared to iFLC peak quantification on SPE by using the tangent scimming method [7].

For statistical analysis, continuous variables were expressed as mean \pm standard (SD) deviation. After One-Sample KolmogorovSmirnov Test, Spearman test were used to determine the correlation, and Wilcoxon Signed Ranks Test were used to determine the difference between the two methods. Passing and Bablok were performed for method comparison that identifies systematic and proportional differences. The intercept of the formula is a measure of the systematic differences between the two methods. The hypothesis of intercept equals to zero is accepted if the confidence interval for intercept contains the value zero. If else, both methods differ at least by a constant amount. The slope of formula is a measure of the proportional differences between the two methods. The hypothesis of the proportional difference is accepted if the confidence interval for slope contains the value 1 . If else, there is at least a proportional difference between the two methods [8]. Bland-Altman plot was used to determine the analysis of the differences. Agreement between methods was realized by computing the concordance correlation coefficient (CCC) [9]. A difference with a p value $<0.05$ was considered significant. Statistical analyses were performed using MedCalc (MedCalc Software, Ostend, Belgium), SPSS 18.0 (SPSS Inc., Chicago, IL, USA) and Microsoft Excel 2016 (Microsoft Corporation, Redmond, WA, USA).

\section{Results}

\section{Method comparison}

$\kappa F L C$ and $\lambda F L C$ were measured in 510 sera using both Sebia FLC and Freelite. Descriptive features of the parameters are shown in Table 1. Parameters were nonparametric distributed. Spearman rank correlation coefficients (CI 95\%) between Sebia and Freelite for $\kappa, \lambda$ and $\kappa / \lambda$ were $r=0.891$ ( 0.866 to 0.916$), 0.774$ ( 0.720 to 0.819$), 0.583$ ( 0.496 to $0.659)$, respectively $(\mathrm{p}<0.0001)$. The CCC of $\kappa$ and $\lambda$ showed a moderate agreement ( $\mathrm{r}=0.68$ and 0.67 , respectively) between both methods. A linear regression analysis of the measured values in a concentration range between 0 and $250 \mathrm{mg} / \mathrm{L}(\mathrm{n}=257)$ which correspond to the measuring ranges of the assays between Sebia and Feelite is shown in Figure 1A. The Passing-Bablok regression line is depicted in Figure 1B. The line demonstrated both proportional and systematic differences between both methods for all parameters. The confidence intervals (CI 95\%) are shown in the graph and the numerical values for intercept and slope were demonstrated in Table 2. In undiluted samples, the Freelite values are more than two times as high as the ELISA values. Over the entire concentration range of the samples measured in the study, the Freelite FLC values were 5.9 fold higher for $\kappa$ FLC samples and 8.6 fold higher for $\lambda$ FLC samples compared to the Sebia FLC assays which indicates that there is no linearity in dilution. Also, Bland-Altman plot revealed that Freelite provides higher values than Sebia especially at the higher levels (Figure 2). The mean biases were $17.2 \mathrm{mg} / \mathrm{L}$ and $1.7 \mathrm{mg} / \mathrm{L}$ for $\kappa$ and $\lambda$, respectively. The difference between methods for $\lambda$ was not significant $(\mathrm{p}=0.184)$ but for $\kappa$ and for the quotient it was significant $(\mathrm{p}<0.05)$.

Table 1. Descriptive properties of free light chains analysed in Sebia and Freelite

\begin{tabular}{|c|c|c|c|c|c|c|c|}
\hline & & Sebia к & Sebia $\lambda$ & Sebia $\kappa / \lambda$ & $\begin{array}{c}\text { Freelite } \\
\kappa\end{array}$ & Freelite $\lambda$ & $\begin{array}{c}\text { Freelite } \\
\kappa / \lambda\end{array}$ \\
\hline \multicolumn{2}{|l|}{$\mathbf{N}$} & 510 & 510 & 510 & 510 & 510 & 510 \\
\hline \multicolumn{2}{|l|}{ Mean } & 30.79 & 42.84 & 2.30 & 79.52 & 124.17 & 12.39 \\
\hline \multicolumn{2}{|l|}{ Median } & 2.49 & 8.06 & 0.48 & 12.15 & 30.26 & 4.92 \\
\hline \multicolumn{2}{|l|}{ Mode } & 19.12 & 20.26 & 0.90 & 27.55 & 18.30 & 1.43 \\
\hline \multicolumn{2}{|c|}{ Std. deviation } & 56.16 & 181.95 & 10.85 & 274.48 & 683.45 & 111.20 \\
\hline \multicolumn{2}{|c|}{ Variance } & 3154 & 33105 & 118 & 75342 & 467108 & 12364 \\
\hline \multicolumn{2}{|l|}{ Skewness } & 8.47 & 12.70 & 13.60 & 11.72 & 8.53 & 16.00 \\
\hline \multicolumn{2}{|l|}{ Kurtosis } & 87.95 & 188.70 & 205.53 & 157.80 & 77.01 & 278.66 \\
\hline \multicolumn{2}{|l|}{ Minimum } & 0.9 & 2.4 & 0 & 1.34 & 0.71 & 0 \\
\hline \multicolumn{2}{|l|}{ Maximum } & 766 & 3137 & 188 & 4070 & 7280 & 2120 \\
\hline \multirow[t]{5}{*}{ Percentiles } & 2.5 & 4.79 & 7.16 & 0.07 & 5.70 & 3.41 & 0.02 \\
\hline & 25 & 13.52 & 15.64 & 0.69 & 17.90 & 12.70 & 1.08 \\
\hline & 50 & 19.12 & 20.26 & 0.90 & 27.55 & 18.30 & 1.43 \\
\hline & 75 & 28.71 & 26.75 & 1.27 & 53.63 & 28.95 & 2.11 \\
\hline & 97.5 & 94.84 & 98.37 & 9.93 & 467.25 & 758.65 & 64.25 \\
\hline
\end{tabular}


Helden VJ (2019) A new free light chain immunoassay shows advantages in the classification and in the follow-up of patients with paraproteinemia compared to a nephelometric assay
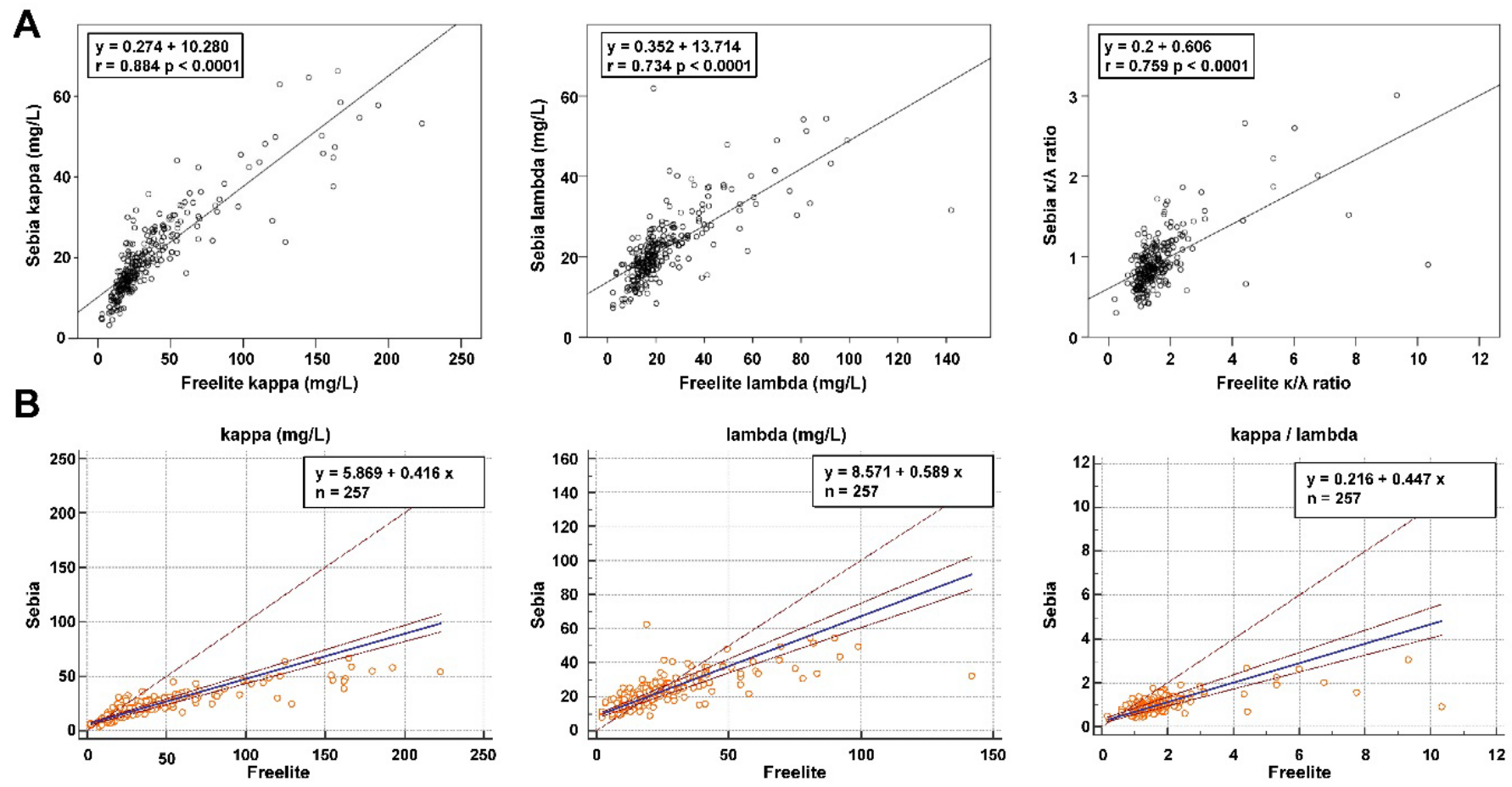

Figure 1. Regression analysis. (A) Linear regression analyses and (B) Passing and Bablok regression analyses of Sebia and Freelite. Scatter diagram with regression line

A

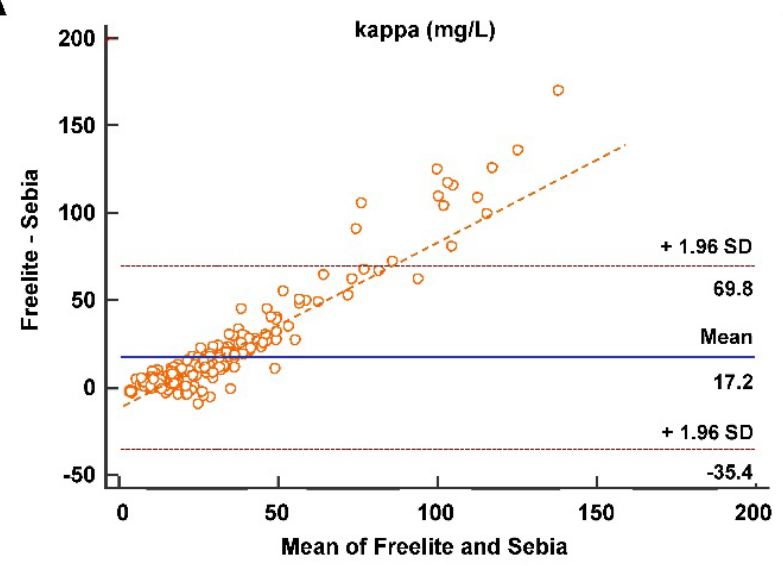

C

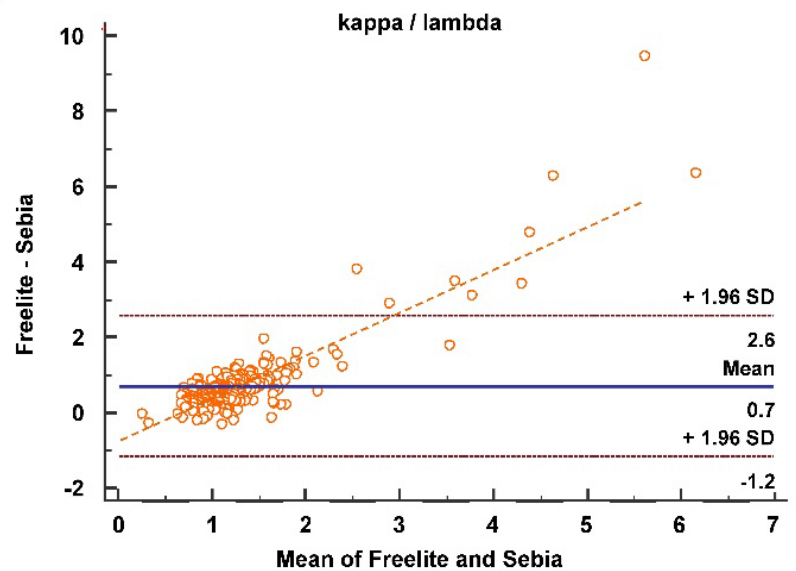

B

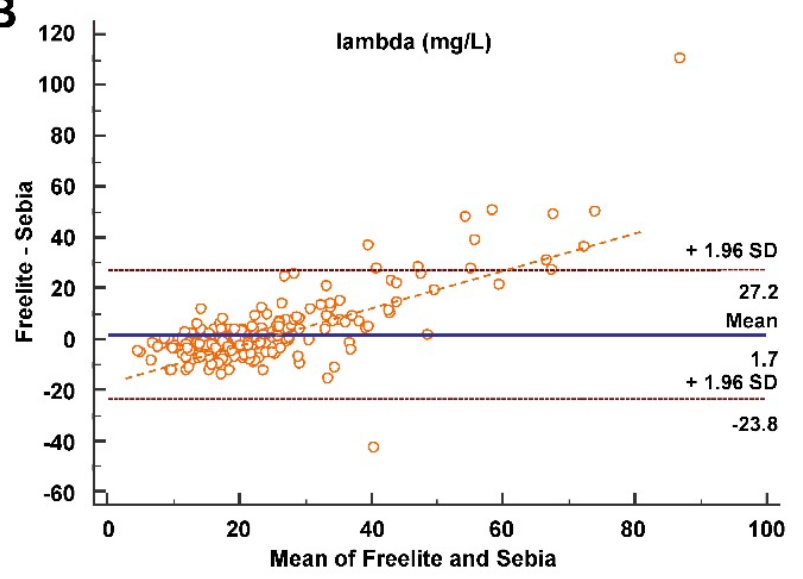

Figure 2. Bland-Altman plots of differences between Sebia and Freelite. Individual plots are shown for (A) the $\kappa$ light chain, (B) the $\lambda$ light chain, and the $\kappa$ to $\lambda$ chain ratios 
Helden VJ (2019) A new free light chain immunoassay shows advantages in the classification and in the follow-up of patients with paraproteinemia compared to a nephelometric assay

Table 2. Passing and Bablok regression of free light chains between Sebia and Freelite

\begin{tabular}{|c|c|c|c|c|}
\hline Light Chain & $\mathbf{r}$ & Formula & $\begin{array}{c}\text { CI 95\% for } \\
\text { intercept }\end{array}$ & $\begin{array}{c}\text { CI 95\% for } \\
\text { slope }\end{array}$ \\
\hline $\boldsymbol{\kappa}$ & 0.893 & $\mathrm{y}=5.94+0.41 \mathrm{x}$ & $4.97-6.78$ & $0.38-0.45$ \\
\hline $\boldsymbol{\lambda}$ & 0.774 & $\mathrm{y}=8.51+0.59 \mathrm{x}$ & $7.28-9.62$ & $0.54-0.65$ \\
\hline $\boldsymbol{\kappa} / \boldsymbol{\lambda}$ & 0.583 & $\mathrm{y}=0.22+0.45 \mathrm{x}$ & $0.14-0.31$ & $0.39-0.51$ \\
\hline
\end{tabular}

Table 3. Comparison of FLC concentration between SPE, Sebia and Freelite

\begin{tabular}{|c|c|c|c|c|}
\hline NO & SPE & Sebia $(\mathrm{mg} / \mathrm{L})$ & Freelite $(\mathrm{mg} / \mathrm{L})$ & FLC \\
\hline 1 & 18,2 & 6,8 & 71,1 & Lambda \\
\hline 2 & 115 & 102 & 11,1 & Kappa \\
\hline 3 & 814 & 687 & 7599 & Lambda \\
\hline 4 & 360 & 324 & 8677 & Lambda \\
\hline 5 & 88 & 79,4 & 7588 & Lambda \\
\hline 6 & 42 & 36,8 & 255 & Kappa \\
\hline 7 & 901 & 860 & 9102 & Lambda \\
\hline 8 & 262 & 272 & 2880 & Lambda \\
\hline 9 & 199 & 201 & 1995 & Lambda \\
\hline 10 & 15,8 & 17,7 & 325 & Kappa \\
\hline 11 & 28,4 & 23,6 & 46,5 & Lambda \\
\hline 12 & 132 & 224 & 1487 & Kappa \\
\hline 13 & 277 & 255 & 2360 & Lambda \\
\hline 14 & 255 & 235 & 4250 & Kappa \\
\hline 15 & 49 & 44,1 & 77,5 & Lambda \\
\hline 16 & 23,4 & 19,5 & 32,4 & Lambda \\
\hline 17 & 82,1 & 67,8 & 166,5 & Kappa \\
\hline 18 & 55,4 & 44,6 & 89,4 & Kappa \\
\hline 19 & 122 & 117 & 227 & Lambda \\
\hline 20 & 399 & 368 & 2471 & Lambda \\
\hline 21 & 1280 & 987 & 4250 & Kappa \\
\hline 22 & 15 & 15,4 & 29,8 & Kappa \\
\hline 23 & 105 & 99,7 & 202 & Lambda \\
\hline 24 & 244 & 223 & 523 & Kappa \\
\hline 25 & 2055 & 1908 & 2258 & Lambda \\
\hline Mean Ratio & $\begin{array}{c}317 \\
1\end{array}$ & $\begin{array}{c}289 \\
0.9\end{array}$ & $\begin{array}{c}3092 \\
10\end{array}$ & \\
\hline
\end{tabular}

The concordance of the clinical interpretation of the FLC ratio between both methods was $76.7 \%$, which resulted in 119 discrepant FLC ratios out of the 510 samples. The moderate agreement was further shown by the Cohen $\kappa$ coefficient of 0.753 . The agreement of the light chain quotients determined with Sebia to the clinical data and to the qualitative findings of the immune fixation was $92.6 \%$, however, higher than that of the quotients determined with Freelite, which was $83.0 \%$. Although, most of the discrepant results were observed in samples with FLC ratio values close to the cut-off value of the reference ranges, at least 6 samples showed results that caused a significant clinical misinterpretation.

\section{Accuracy of FLC measurements}

Differences between Freelite and Sebia FLC were most apparent at the high end of the concentration range. To assess the accuracy of both of the FLC assays results were compared to SPE concentrations in 25 patients with measurable sFLC peaks on SPE. The concentrations of the iFLC in the 25 serum samples ranged from 6.8 to $22,580 \mathrm{mg} / \mathrm{L}$. The Freelite sFLC concentrations were consistently higher in 23 of the 25 samples tested with a mean 10 -fold overestimation compared to SPE. The complete data of the comparison are displayed in Table 3. The observed differences were unsystematic and patient-dependent. Sebia sFLC concentrations showed a good comparability to the SPE FLC peak concentrations with an average 0.9 -fold underestimation. Figure 3 shows four examples of these discrepant samples. Beside the SPE and the IFE the FLC determination with Sebia and Freelite are displayed. As can be seen in the figure, contradictory statements are made about the two methods in the four examples. In example $A$ of a free $\boldsymbol{\lambda}$ light chain, the Sebia value clearly fits better to the peak height of the paraprotein in the SPE which was $4250 \mathrm{mg} / \mathrm{L}$. In example B (IgA $/ \mathrm{Gr})$ the opposite is observed. Here the value determined with Freelite appears too low for the peak height in the SPE. A quantification was not possible because of the biclonality. In C, IgA/ $\lambda$,. only the Sebia ELISA shows an increased value and a decreased ratio. $\mathrm{D}$ is a putative positive $\lambda$ FLC. The Sebia ELISA value is here only slightly increased, while the free elite value is 11 times higher, although in the SPE no quantifiable extra gradient and in the immune fixation no sharply defined band is recognizable.

\section{Reruns}

Serum FLC covers a wide concentration range that must be covered by analytical methods. Often serum samples with higher, but
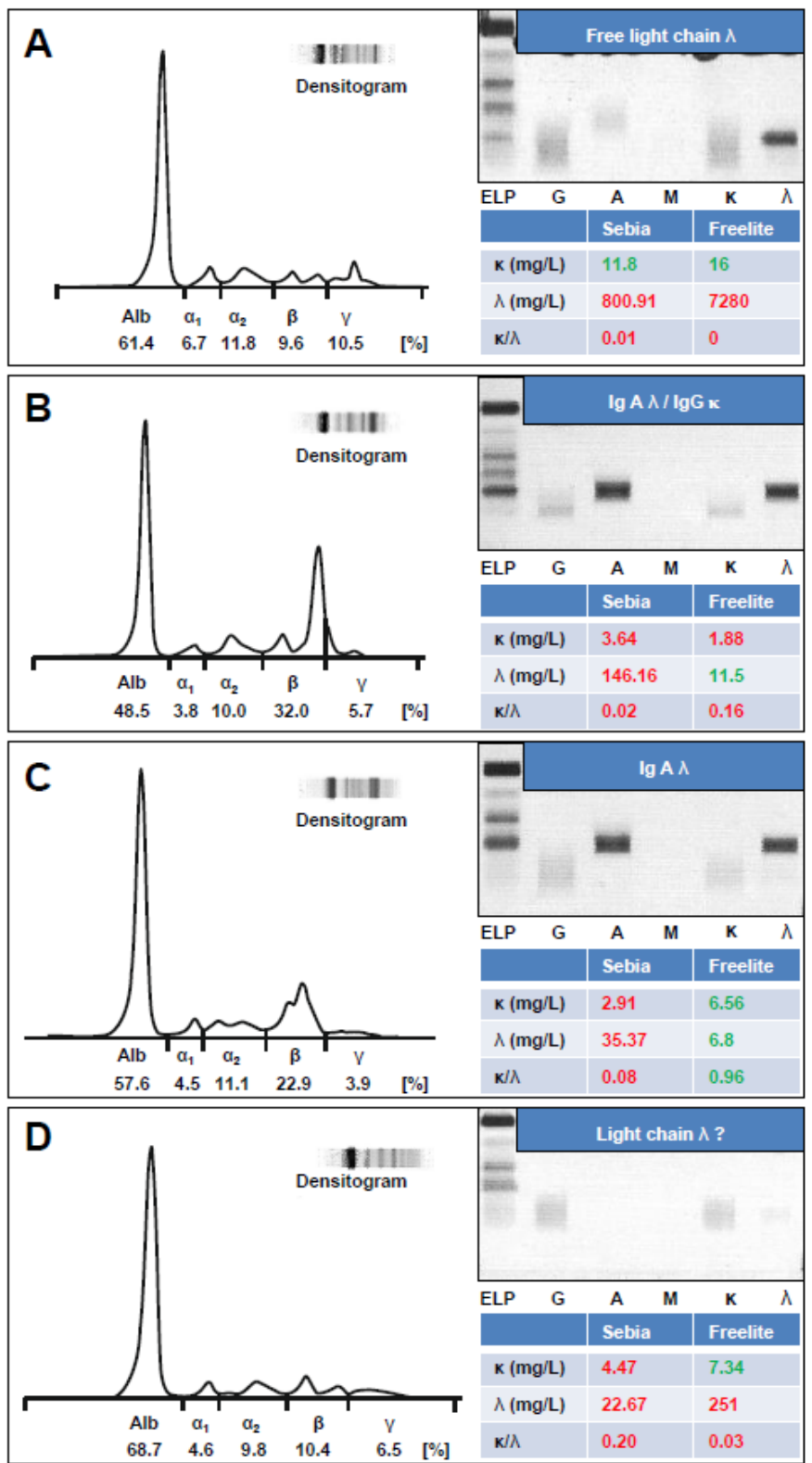

Figure 3. Exemplary presentation of discrepant samples 
also with lower FLC concentrations have to be re-analyzed several times before a valid result is obtained using the common turbidimetric or nephelometric methods. To measure the 510 serum samples of this study, a total of 1234 dilution runs were necessary using the Freelite reagent on the BNII nephelometer. This averages 2.34 approaches to determine a $\kappa / \lambda$ ratio. Using the Sebia FLC ELISA, 1067 approaches were sufficient, which corresponds to a ratio of 1:2.08 per reported result.

\section{Discussion}

This retrospective study describes under routine conditions the performance of the Sebia FLC assay for the quantitative determination of $\kappa F L C$ and $\lambda F L C$ in serum. The measurement of monoclonal free light chains is an essential supplementary test in the screening of patients with suspected monoclonal gammopathy, in the course of prognostic stratification and within therapy monitoring $[1,10,11]$. We report here on this modern sandwich ELISA, which uses polyclonal antibodies as a modern new-fashioned platform for the sensitive, accurate and reproducible quantification of serum FLC.

In principle, it is difficult to compare the two methods as there is neither a reference method nor a certified reference material available. Despite a particularly good agreement, in individual cases, especially within high FLC concentrations, there are particularly significant, clinically relevant deviations in addition to the two methods that can also be demonstrated under consideration of the respective reference ranges (Figure 4). In the majority of cases, the sFLC concentrations determined with the ELISA fit better to the concentration to the concentration of the $\mathrm{M}$ protein fraction determined by integration of the peak in the SPE.

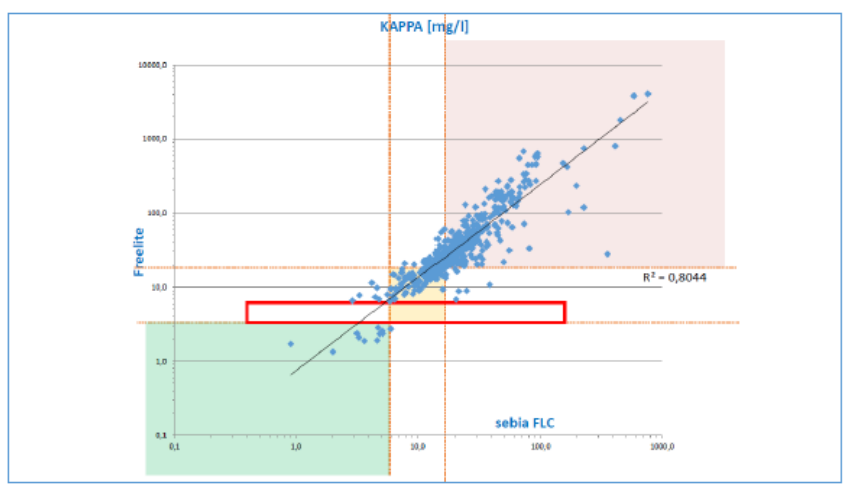

Laboratory methods for FLC screening have traditionally used electrophoresis and immunofixation of urinary proteins because they are more sensitive than SPE and IFE in serum. The sensitivity of serum FLC measurements was significantly increased by use of automated nephelometric assays [12]. So that the calculation of the ratio FLC $\kappa / \lambda$ has become a sensitive method for the detection and the monitoring of monoclonal gammopathies. Meanwhile, alternative FLC immunoassays are available on differentiated analysis platforms for diagnostic laboratories. Because of the heterogeneity of FLC [13-15], any FLC test has specific analytical limitations. The three currently routinely available assays show significant differences in value levels in method comparisons [16,17], especially depending on the antibodies used. These methods show significant differences in the type of antibodies used. The advantage of using polyclonal antibodies in comparison to monoclonal antibodies is the detection of a larger range of epitopes, which results in tests using a polyclonal reagent having a higher detection rate of monoclonal FLC [18]. On the other hand, monoclonal reagents have been reported to be more suitable for follow-up of individual patients due to their higher specificity and reproducibility. However, both test methods show analytical limitations regarding linearity and precision.

Previous comparative studies between Freelite and N Latex FLC assays showed significant differences especially at high FLC concentrations $[19,20]$. It has been shown that this overestimination is caused by polymerization of the FLC in Freelite as well as N Latex nephelometric assays $[19,21]$. The results of our study may be due to such polymerizations. FLC determination was consistently 10fold higher than the values quantitated from electrophoresis. In contrast, the values of the FLC ELISA are significantly lower and

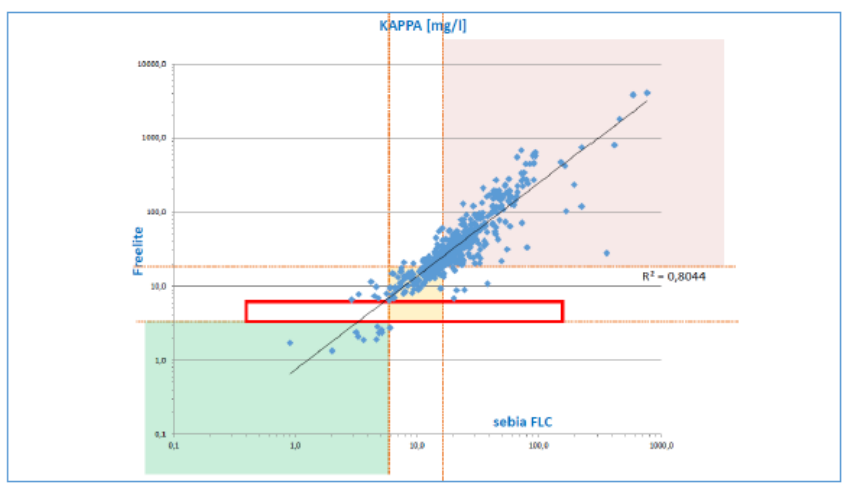

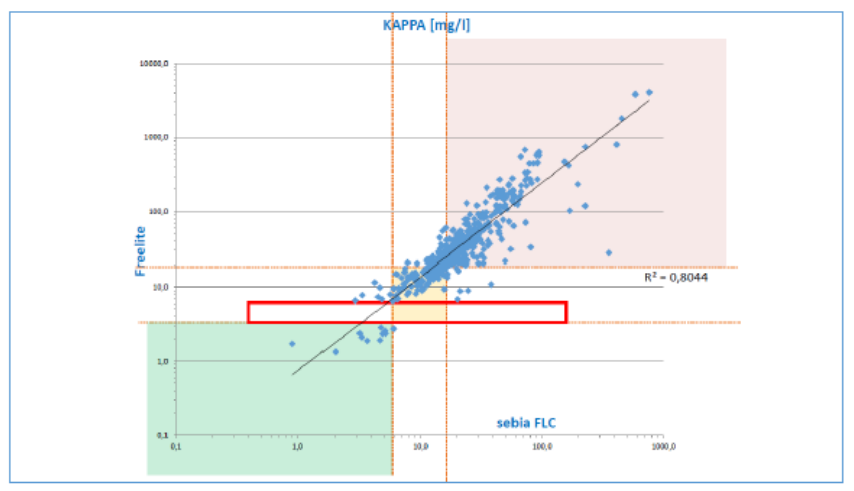

Figure 4. Deviations between the different methods in relation to the respective reference ranges 
Helden VJ (2019) A new free light chain immunoassay shows advantages in the classification and in the follow-up of patients with paraproteinemia compared to a nephelometric assay

much more consistent with those obtained by quantification via electrophoresis especially in samples with very high FLC titers. This strong overestimation of samples with high FLC concentrations by the Freelite assay is in accordance with the previously published facts and suggests that it is due to FLC polymerization [21,22]. Due to the completely different values of both assays, it is not possible to use both tests alternately in the follow-up of a patient. Therefore, if a laboratory wishes to switch to the new ELISA technology for the determination of FLC, it is urgently necessary to perform both methods in parallel for a certain period of time in order to gain an impression of each patient's new individual value situation.

Serum FLC concentrations can extend over a wide range (from 1 to $100,000 \mathrm{mg} / \mathrm{L}$ ), depending on the extent of the underlying disease, and are therefore susceptible to excess antigen that can lead to a high dose hook effect [23]. The suitability of the non-ELISA FLC assays is additionally limited by the lack of dilution linearity for samples with high FLC titers [24,25]. Finally, due to the limited measurement range of today's FLC assays, a large number of dilutions are often necessary to determine a final valid patient result [26]. The new Sebia FLC ELISAs are not subject to these restrictions, they are linear in dilution and are not subject to antigen excess [26-28]. They have good reproducibility and good lot to lot stability [6]. Since they also require fewer dilution steps, they are a alternative to the Freelite FLC assay for monitoring patients with monoclonal gammopathy. As the current guidelines provide for the use of the current turbidimetric or nephelometric assays [3], the inclusion of ELISA technology in future versions should be considered as the results of ELISA determinations are in better agreement to those obtained by the SPE and immunofixation reference tests.

In conclusion, the Sebia FLC assays offer a robust platform for sensitive and accurate sFLC measurements. Sebia FLC showed better agreement with SPE FLC peak concentrations. The Sebia FLC results are in line with the quantification of M-Protein in the SPE and the Sebia FLC repeat rate is lower due to the larger measurement range. For these reasons, the Sebia FLC assay is presently the superior method for the determination of free light chains in serum.

\section{Funding}

None declared

\section{Conflicts of Interest}

The authors declare no conflict of interests.

\section{References}

1. Dispenzieri A, Kyle R, Merlini G, Miguel JS, Ludwig H, et al. (2009) International Myeloma Working Group guidelines for serum-free light chain analysis in multiple myeloma and related disorders. Leukemia 23: 215-224.

2. Katzmann JA, Clark RJ, Abraham RS, Bryant S, Lymp JF et al. (2002) Serum reference intervals and diagnostic ranges for free kappa and free lambda immunoglobulin light chains: relative sensitivity for detection of monoclonal light chains. Clin Chem 48: 1437-1444.

3. Moreau P, San Miguel J, Sonneveld P, Mateos MV, Zamagni E, et al. (2017) Multiple myeloma: ESMO Clinical Practice Guidelines for diagnosis, treatment and follow-up. Ann Oncology 28: 52-61.

4. Campbell JP, Cobbold M, Wang Y, Goodall M, Bonney SL, et al. (2013) Development of a highly-sensitive multi-plex assay using monoclonal antibodies for simultaneous measurement of kappa and lambda immunoglobulin free light chains in serum and urine. J Immunol Meth 391: 1-13.

5. Bradwell AR, Carr-Smith HD, Mead GP, Harvey TC, Drayson MT (2003) Serum test for assessment of patients with Bence Jones myeloma. Lancet 361: 489-491. [Crossref]
6. Jacobs JFM, de Kat Angelino CM, Brouwers HMLM, Croockewit SA, Joosten I, et al. (2018) Evaluation of a new free light chain ELISA assay: bringing coherence with electrophoretic methods. Clin Chem Lab Med 56: 312-322.

7. Schild C, Wermuth B, Trapp-Chiappini D, Egger F, Nuoffer M (2008) Reliability of $\mathrm{M}$ protein quantification: comparison of twopeak integration methods on Capillarys 2. Clin Chem Lab Med 46:846-847.

8. Passing H, Bablok W (1983) A new biometrical procedure for testing the equality of measurements from two different analytical methods. Application of linear regression procedures for method comparison studies in clinical chemistry, Part I. J Clin Chem Clin Biochem 21: 709-720.

9. Lin LI (1989) A concordance correlation coefficient to evaluate reproducibility Biometrics 45: 255-268.

10. Graziani MS Merlini G (2014) Serum free light chain analysis in the diagnosis and management of multiple myeloma and related conditions. Expert Rev Mol Diagn 23: 55-66.

11. Raikumar SV, Dimopoulos MA, Palumbo A, Blade J Merlini G, Mateos, et al. (2014) International Myeloma Working Group updated criteria for the diagnosis of multiple myeloma. Lancet Oncol 15: 538-548.

12. Bradwell AR, Carr-Smith HD, Mead GP, Tang LX, Showell PJ, et al. (2001). Highly sensitive, automated immunoassay for immunoglobulin free light chains in serum and urine. Clin Chem 47: 673-680

13. te Velthuis H, Knop I, Stam P, van den Broek M, Bos HK, et al. (2011) N Latex FLC - new monoclonal high-performance assays for the determination of free light chain kappa and lambda. Clin Chem Lab Med 49: 1323-1332. [Crossref]

14. Campbell JP, Heaney JL, Shemar M, Baldwin D, Griffin AE, et al. (2017) Developmen of a rapid and quantitative lateral flow assay for the simultaneous measurement of serum kappa and lambda immunoglobulin free light chains (FLC): inception of a new near patient FLC screening tool. Clin Chem Lab Med 55: 424-434.

15. Tate J, Bazeley S, Sykes S, Mollee P (2009) Quantitative serum free light chain assay-analytical issues. Clin Biochem Rev 30: 131-140.

16. Jacobs JF, Tate JR, Merlini G (2016) Is accuracy of serum free light chain measurement achievable? Clin Chem Lab Med 54: 1021-1030. [Crossref]

17. VanDuijn MM, Jacobs JF, Wevers RA, Engelke UF, Joosten I, et al. (2015) Quantitative measurement of immunoglobulins and free light chains using mass spectrometry. Anal Chem 87: 8268-8274. [Crossref]

18. Graziani MS (2016) Measurement of free light chains - pros and cons of current methods. Clin Chem Lab Med 54: 1015-1020. [Crossref]

19. Di Noto G, Cimpoies E, Dossi A, Paolini L, Radeghieri A, et al. (2015) Polyclona versus monoclonal immunoglobulin-free light chains quantification. Ann Clin Biochem 52: $327-336$.

20. Kim HS, Kim HS, Shin KS, Song W, Kim HJ, et al. (2014) Clinical comparisons of two free light chain assays to immunofixation electrophoresis for detecting monoclonal gammopathy. Biomed Res Int 2014: 647238.

21. de Kat Angelino CM, Raymakers R, Teunesen MA, Jacobs JF, Klasen IS (2010) Overestimation of serum kappa free light chain concentration by immunonephelometry. Clin Chem 56: 1188-1190. [Crossref]

22. Abraham RS, Charlesworth MC, Owen BA, Benson LM, Katzmann JA, et al. (2002) Trimolecular complexes of lambda light chain dimers in serum of a patient with multiple myeloma. Clin Chem 48: 1805-1811. [Crossref]

23. Murata K, Clark RJ, Lockington KS, Tostrud LJ, Greipp PR, et al. (2010) Sharply increased serum free light-chain concentrations after treatment for multiple myeloma. Clin Chem 56: 16-18.

24. Jacobs JF, Hoedemakers RM, Teunissen E, van der Molen RG, te Velthuis H. (2012) Effect of sample dilution on two free light chain nephelometric assays. Clin Chim Acto 413: 1708-1709.

25. Vercammen M, Meirlaen P, Broodtaerts L, Van de Broek I, Bossuyt X (2011) Effect of sample dilution on serum free light chain concentration by immunonephelometric assay. Clin Chim Acta 412: 1798-1804.

26. Ialongo C, Pieri M, Bernardini S (2017) Smart management of sample dilution using an artificial neural network to achieve streamlined processes and saving resources: the automated nephelometric testing of serum free light chain as case study. Clin Chem Lab Med 55: 231-236. 
Helden VJ (2019) A new free light chain immunoassay shows advantages in the classification and in the follow-up of patients with paraproteinemia compared to a nephelometric assay

27. Lutteri L, Aldenhoff MC, Cavalier E (2018) Evaluation of the new Sebia free light chain assay using the AP22 ELITE instrument. Clin Chim Acta 487: 161-167. [Crossref]
28. Pekar JD, Schraen S, Grzych G, Manier S, Oenrad B (2019) Antigen excess pitfall for free light chains measurements solved by ELISA assay. Am J Hematol 94: E120-E122.

Copyright: (C2019 Helden VJ. This is an open-access article distributed under the terms of the Creative Commons Attribution License, which permits unrestricted use, distribution, and reproduction in any medium, provided the original author and source are credited. 